\title{
Instantiating the Contingent Bids Model of Truthful Interdependent Value Auctions
}

\section{Citation}

Ito, Takayuki, and David C. Parkes. 2006. Instantiating the contingent bids model of truthful interdependent value auctions. In Proceedings of the fifth International Joint Conference on Autonomous Agents and Multiagent Systems: May 8-12, 2006, Hakdate, Japan, ed. H. Nakashima, M. P Wellman, G. Weiss, P. Stone, ACM Special Interest Group on Artificial Intelligence, 1151-1158. New York, N.Y.: ACM Press.

\section{Published Version}

doi:10.1145/1160633.1160842

\section{Permanent link}

http://nrs.harvard.edu/urn-3:HUL.InstRepos:3996852

\section{Terms of Use}

This article was downloaded from Harvard University's DASH repository, and is made available under the terms and conditions applicable to Other Posted Material, as set forth at http:// nrs.harvard.edu/urn-3:HUL.InstRepos:dash.current.terms-of-use\#LAA

\section{Share Your Story}

The Harvard community has made this article openly available.

Please share how this access benefits you. Submit a story.

Accessibility 


\section{Instantiating the Contingent Bids Model of Truthful Interdependent Value Auctions}

\author{
Takayuki Ito \\ Center for Coordination Science, \\ Sloan School of Management, \\ Massachusetts Institute of Technology \\ 3 Cambridge Center, NE20-336 \\ Cambridge, MA 02142 \\ takayuki@mit.edu
}

\author{
David C. Parkes \\ Division of Engineering and Applied Sciences, \\ Harvard University, \\ Cambridge MA 02138 USA. \\ parkes@eecs.harvard.edu
}

\begin{abstract}
We consider the problem of auction design with agents that have interdependent values, i.e. values that depend on each others' private signals. We adopt the contingent bids model of Dasgupta and Maskin [3], and allow agents to submit bids of the form "if player 1 bids \$x for good $A$ then I will bid \$y." Our main contribution is to identify a specific linear valuation model for which there exists an efficient auction for a single item, and then extend this to provide an approximately efficient combinatorial auction with single-minded bidders. In both auction, winners and payments are computed from the fixed point of the valuation mapping defined by contingent bids. We also adopt search in order to construct a variation on the singleitem auction with improved revenue. In closing, we discuss the (many) challenges in moving to more general models of interdependent valuations.
\end{abstract}

\section{INTRODUCTION}

We consider the problem of auction design with agents that have interdependent values. In this setting, the value of an allocation can depend on the private signals of the other agents.

Allocation problems with interdependent values often arise in auction settings. Consider, for example, the sale of a case of vintage wine to a group of buyers with distributed information about the actual quality (and thus value) of the wine. Some of the bidders may have tasted similar wines, some may have read reviews, while still others may be complete novices. Interdependent values can also arise when a bidder's value for an allocation depends on the details of the allocation of goods to other bidders, and thus its value depends (indirectly) on the values of other bidders. Consider an auction for wireless spectrum. A wireless company might assign more value to some bundle of licenses if non-competing companies (e.g. with different business models) win the remaining licenses.

\footnotetext{
*Visiting from Nagoya Institute of Technology, Japan
}

Permission to make digital or hard copies of all or part of this work for personal or classroom use is granted without fee provided that copies are not made or distributed for profit or commercial advantage and that copies bear this notice and the full citation on the first page. To copy otherwise, to republish, to post on servers or to redistribute to lists, requires prior specific permission and/or a fee.

AAMAS'06 May 8-12 2006, Hakodate, Hokkaido, Japan.

Copyright 2006 ACM 1-59593-303-4/06/0005 ...\$5.00.
In addition to settings of electronic commerce, we believe that interdependent value models will be useful for many coordination problems in multiagent systems. Consider, for example decision making about resource allocation on computational grids, for instance in allowing a team of scientists to evaluate their value for receiving the right to be the first to work with a new data set. Accurately determining this value may require aggregating the private information of a number of different teams.

The design of efficient (and optimal) auctions with interdependent values has received some attention in the economic theory literature. In particular, under the assumption that the private information (signal) of a bidder can be captured by a single number (in combination with monotonicity requirements that prevent arbitrary information from being encoded in this number), then there exist ex post efficient auctions [10]. For more general models with multi-dimensional signals there are strong negative results about the ability to implement efficient outcomes (or even much at all!) in an equilibrium $[3,8]$.

We too will work within the one-dimensional signal model. Specifically, we follow Dasgupta and Maskin [3], but depart from the other literature on interdependent value models, by working with contingent bids. As we discuss in the next section, the alternative is to work in a direct revelation framework, but this appears much more cumbersome for bidders. With contingent bids, a bidder can state information such as "my bid value is $\$ \mathrm{x}$ when bidder 2's value is \$y." Bidders do not need to describe either their own signal to the mechanism, nor the domain of signals for the other bidders. We agree with Dasgupta and Maskin that this seems very difficult. In our wine example, all bidders would have to agree with the auctioneer on a language to talk about the quality of wine. In our grid computing example, all research groups would have to agree on a common language to discuss the potential value of some new data. In this aspect, our focus on contingent bids differentiates this work from the earlier work in distributed AI of Dash et al. [4] and Ito et al. [5, 7], who consider direct revelation mechanisms.

We adopt contingent bids as first-order representations of agents' private valuation models. Our main contribution is to provide a specific instantiation of Dasgupta and Maskin's [3] contingent bid model. In particular, we define a linear contingent-bid language and establish necessary and sufficient conditions for bids to satisfy the technical conditions required for the existence of an efficient auction. We first introduce this for the single item allocation problem and later extend the language to allow for combinatorial auctions with single minded bidders. A single minded bidder is interested in just one particular bundle of goods. 
In pursuit of an auction with improved revenue properties, for instance of importance to a seller of vintage wine, we then generalize the auction to include a "dummy" agent that represents the interest of the seller and submits a bid to increase competition and also to have the effect of setting a reservation price. Following the ideas introduced in Likhodedov and Sandholm [12, 13] we then use search to identify an auction with better revenue properties within this class of generalized auctions. Unlike the existing theory on optimal auctions with interdependent values [2], in which interim individual rationality (IR) is provided, ${ }^{1}$ our auctions have the more reasonable property of ex post IR. This means that an agent will never pay more than its value for an allocation.

In closing, we discuss the many challenges in moving towards more general models of interdependent valuations. Even within the setting of one-dimensional signals we identify a significant practical problem in extending multi-unit auctions to allow for more than single-minded bidders. The problem relates to the amount of information that bidders are required to report to an auction about the possible valuations of other bidders.

\subsection{Related Work}

Dash et al. [4] define a multi-item variant on the efficient, directrevelation mechanism for multiple identical items in Krishna [10]. Unlike the model of Dasgupta and Maskin [3] (DM), which we adopt in this paper, the model of Dash et al. requires that the mechanism knows the valuation function and signal domains of each bidder. Bidders report their signal and the mechanism determines the outcome and payments. Ito and colleagues $[5,6,7]$ also study a model with one-dimensional signals in the context of a multiitem allocation problem. Unlike Dash et al. [4], their mechanisms do not need to know the valuation functions of agents. However, they remain direct revelation schemes, in that agents bid both valuation functions and signals. Again, the language for describing an agent's signal must be common knowledge to all agents and the mechanism. Ito and colleagues assumed special structure to the problem, namely that the interdependency is acyclic: there are "experts" whose signal affect the values of the other agents ("amateurs"). The experts' values are unaffected by the signal of any amateur and no amateur's signal affects the value of any other amateur. This allows for positive results in their model, which is otherwise quite general.

Jehiel et al. [9] and earlier Jehiel and Moldovanu [8], provide strong limits on ex post Nash implementation with interdependent valuations. When signals are multi-dimensional then efficiency is generally impossible, and it can be the case that no interesting social choice function can be implemented. Although, see Bikhchandani [1] for a discussion of when this negative result breaks down (and implementation is again possible.) In grid computing, an example of a multi-dimensional signal is when an agent has two signals, one relates to the novelty of the data set and one relates to the noise in the data set. One cannot combine these into one dimension while retaining properties such as increasing value with signal, also required for ex post implementation. Multidimensional signals present a new challenge, unseen in private value settings, because there are typically multiple signal values that correspond to the same value on some bundle of goods and no good way to provide incentives for an agent to report the correct pair of signals. Reports of the correct pair of signals are important to allow other

${ }^{1}$ Cremer and McLean [2] show that if there is even a small amount of correlation between agent signals then the seller can extract all of the surplus from the participants. However, this requires interim IR. We are not aware of any revenue optimality results for the more reasonable requirement of ex post IR. agents to learn their correct value.

Finally, work on two-stage mechanisms [16] and other ascending price designs (see Krishna [10]) also shares the concern of DM for limiting the knowledge required of the mechanism and agents. We are not aware of previous attempts to instantiate the contingent bid model of DM, as we do in this paper.

\section{PRELIMINARIES}

Consider a single item problem. Each agent $i \in\{1, \ldots, N\}$ has a signal $s_{i} \in S_{i}$ from some signal space $S_{i}$. The value $z_{i}(s) \geq 0$ of agent $i$ for the item depends on the joint signals $s=\left(s_{1}, \ldots, s_{N}\right){ }^{2}$ The efficient allocation is to allocate the item to the agent with maximal value. Assume quasilinear utilities, with utility $z_{i}(s)-p$ for the item at price $p$. Throughout the paper, vector notation $s_{-i}$ denotes $\left(s_{1}, \ldots, s_{i-1}, s_{i+1}, \ldots, s_{N}\right)$.

First suppose that the auctioneer has knowledge of valuation functions $\left(z_{1}, \ldots, z_{N}\right)$, but not signals. One can construct a truthful and efficient "second-price" auction for some technical restrictions as follows. Each agent reports its signal $\hat{s}_{i}$, perhaps untruthfully. The auctioneer computes $z_{i}(\hat{s})$ for every $i$, allocates the item to the agent with $\max _{i}\left\{z_{i}(\hat{s})\right\}$ and then charges the agent $p_{i}=$

$$
\begin{array}{ll}
\min _{s_{i}^{\prime}} & z_{i}\left(s_{i}^{\prime}, \hat{s}_{-i}\right) \\
\text { s.t. } & z_{i}\left(s_{i}^{\prime}, \hat{s}_{-i}\right) \geq \max _{j \neq i}\left\{z_{j}\left(s_{i}^{\prime}, \hat{s}_{-i}\right)\right\}
\end{array}
$$

The auction is efficient if valuations satisfy monotonicity,

$$
\frac{\partial z_{i}(s)}{\partial s_{i}} \geq 0, \quad \forall s, \forall i
$$

and the single-crossing condition (SCC),

$$
\frac{\partial z_{i}(s)}{\partial s_{i}}>\frac{\partial z_{j}(s)}{\partial s_{i}}
$$

for all $s$ such that $z_{i}(s)=\max _{j \neq i}\left\{z_{j}(s)\right\}$.

THEOREM 1. [10] The second-price auction is efficient when the monotonicity and single-crossing property holds.

PROOF. (sketch) Each agent $i$ faces a price $p_{i}$ that solves Eq. (1) and is independent of its own report. Suppose the other agents bid truthfully, with $\hat{s}_{-i}=s_{-i}$. Then, by SCC and monotonicity, if agent $i$ bids truthfully $\left(\hat{s}_{i}=s_{i}\right)$ then it is allocated the good whenever its value is greater than $p_{i}$, and not allocated the good otherwise. (Fix $s_{-i}$. Then SCC implies that for all signals less than that which defines $p_{i}$ agent $i$ is not a winner, and for all signals greater agent $i$ is the winner.) By reporting its true signal, the agent maximizes its utility with respect to a price that is independent of its own report. This is an ex post Nash equilibrium (but not dominant strategy equilibrium). Truthful reporting is only best-response when the other agents are also truthful (but whatever their true signals), because the reports of the other agents are required for agent $i$ 's true value to be correctly determined.

This auction reduces to the Vickrey auction for private value models. Yet, there is a practical problem with this protocol. As argued by DM, the auctioneer must know the signal space of agents (in order to define an expressive language for signals), and also the

\footnotetext{
${ }^{2}$ Notation $z_{i}: S_{1} \times \ldots S_{N} \rightarrow \mathbb{R}_{\geq 0}$ denotes an agent's value as a function of the signals of all agents. Below, $b_{i}: \mathbb{R}_{\geq 0}^{N-1} \rightarrow \mathbb{R}_{\geq 0}$ denotes a contingent bid function, and $v_{i} \in \mathbb{R}_{>0}$ is reserved to denote the value of agent $i$ : as determined either via $z_{i}(s)$ or as the fixed point defined by agents' contingent bids.
} 
valuation function $z_{i}$ of each agent. This seems to be a heavy burden. Moreover, each agent must report its signal. In general, a signal (for instance information about a case of wine, or the data set in grid computing) can be difficult for a participant to describe.

Instead, DM work in the framework of contingent bids. Let $v_{i}=z_{i}(s) \geq 0$ denote agent $i$ 's value for the good. Agents submit contingent bids, $b_{i}: \mathbb{R}_{\geq 0}^{N-1}$, where $b_{i}\left(v_{-i}\right)$ is the (reported) value given values $v_{-i}=\left(v_{1}, \ldots, v_{i-1}, v_{i+1}, v_{N}\right)$ of other agents. Given contingent bids, the auctioneer determines reported values as the fixed point of the mapping:

$$
\left(v_{1}, \ldots, v_{N}\right) \longmapsto\left(b_{1}\left(v_{-1}\right), \ldots, b_{N}\left(v_{-N}\right)\right)
$$

We refer to this fixed point as a valuation equilibrium, and denote the fixed point as $v^{\circ}$. Agent $i$ is said to bid truthfully when $v^{\circ}$ corresponds to interdependent valuations $z(s)$ given signals, i.e.

$$
b_{i}\left(z_{-i}\left(s_{i}, s_{-i}^{\prime}\right)\right)=z_{i}\left(s_{i}, s_{-i}^{\prime}\right), \quad \forall s_{-i}^{\prime}, \forall s_{i}
$$

Each agent submits a contingent bid, so that its value in the valuation equilibrium is equal to its true value given the signal information $s_{-i}$ that is implicit in the fixed-point values of the other agents. When all agents bid truthfully the fixed point $\left(v_{1}^{\circ}, \ldots, v_{N}^{\circ}\right)=$ $\left(z_{1}(s), \ldots, z_{N}(s)\right)$.

DM propose the following contingent bid auction, which is well defined when there exists a unique valuation equilibrium:

1. Given bids, compute the valuation equilibrium $\left(v_{1}^{\circ}, \ldots, v_{N}^{\circ}\right)$.

2. Allocate the item to the agent with the maximal fixed-point value, breaking ties at random.

3. For the winner, agent $i$, compute payment $p_{i}$ as $\min v_{i}^{\prime}$ s.t. $v_{i}^{\prime} \geq \max _{j \neq i}\left\{v_{j}^{*}\right\}$, where $v_{j}^{*}=b_{j}\left(v_{i}^{\prime}, v_{-i j}^{*}\right)$ for all $j \neq$ $i$, denoting the fixed-point valuations to other agents when agent $i$ 's bid is adjusted to some value $v_{i}^{\prime}$.

The payment to the winner is computed as the minimal (noncontingent) value that the winner could have submitted and still remained the winner.

THEOREM 2. [3] The contingent-bid auction is efficient (in an ex post Nash equilibrium) when valuations satisfy monotonicity and SCC, and when the fixed point of the mapping defined by contingent bids is unique.

The proof follows (modulo technicalities about the uniqueness of fixed points) from the structure of the proof of Theorem 1. Agents will bid truthfully in equilibrium.

Contingent bids make interdependent value auctions practical. The mechanism does not need to know the signal spaces or valuation functions of agents. On the other hand, DM note that the bidders must now have knowledge of each others' valuation functions to be able to deduce implicit signal information in the valuation equilibrium. ${ }^{3}$ We adopt a different view on this issue. Let us simply consider the contingent-value model as the underlying model of value interdependency. With this view point, the contingent bid model requires an agent to know enough about other agents (and that this is known by other agents and so on ad infinitum) to define its value contingent on their values.

\footnotetext{
${ }^{3} \mathrm{In}$ fact, DM remark that all that is necessary is that the agents are able to make correct inferences at the inflection point that defines the price, i.e. where the winner changes from one agent to another agent.
}

\section{A LINEAR VALUATION MODEL}

An actual instantiation of the contingent-bid auction requires a concrete bidding language, designed so that bids submitted in the language define a mapping (Eq. 4) with a unique fixed point, and so that valuations at this fixed point satisfy monotonicity and SCC. In this paper we adopt a linear bidding language,

$$
b_{i}\left(v_{-i}\right)=v_{i 0}+\sum_{j \neq i} \alpha_{i j} v_{j}
$$

where $v_{i 0} \geq 0$ denotes agent $i$ 's stand-alone value when other agents have zero value, and $\alpha_{i j} \in[0,1)$ is a weight assigned by agent $i$ to the value of other bidders. Conceptually, the stand-alone value $v_{i 0}$ plays the role of the signal and the $\alpha$-weights indicate implicit structure about the relationships between valuation functions $z=\left(z_{1}, \ldots, z_{N}\right)$ in the economy.

\subsection{Example}

Suppose there are three companies, $\{1,2,3\}$, bidding in an auction. Their values are interdependent. For example, company 1 is paying attention to the values of company 2 and company 3 :

$$
b_{1}\left(v_{2}, v_{3}\right)=50+0.3 v_{2}+0.5 v_{3}
$$

In Eq. (7), company 1 places the weight 0.3 on the value of company 2 and weight 0.5 on the value of company 3 . Similarly, we assume 2 and 3 have the following (truthful) contingent bids:

$$
\begin{aligned}
& b_{2}\left(v_{1}, v_{3}\right)=60+0.4 v_{1}+0.4 v_{3} \\
& b_{3}\left(v_{1}, v_{2}\right)=70
\end{aligned}
$$

Namely, 1 and 2 are paying attention to the values of the other companies, while 3 ignores the others' values. The sum of weights are less than 1.0, which ensures uniqueness of the valuation equilibrium (the details of this result are shown in Section 3.2.) The valuations implied by these bids are computed as the fixed point $\left(v_{1}^{\circ}, v_{2}^{\circ}, v_{3}^{\circ}\right)$ of mapping Eq. (4). In this case, the fixed point can be found by solving:

$$
\begin{aligned}
& v_{1}^{\circ}=50+0.3 v_{2}^{\circ}+0.5 v_{3}^{\circ} \\
& v_{2}^{\circ}=60+0.4 v_{1}^{\circ}+0.4 v_{3}^{\circ} \\
& v_{3}^{\circ}=70,
\end{aligned}
$$

for $\left(v_{1}^{\circ}, v_{2}^{\circ}, v_{3}^{\circ}\right)=(126.6,138.6,70.0)$. Because at the fixed point, company 2 has the maximum value it is the winner. Its payment is computed as $v_{2}^{\prime}$ that solves $v_{2}^{\prime}=\max \left\{v_{1}^{*}, v_{3}^{*}\right\}$, where $v_{1}^{*}=b_{1}\left(v_{2}^{\prime}, v_{3}^{*}\right)$ and $v_{3}^{*}=b_{3}\left(v_{2}^{\prime}, v_{1}^{*}\right)$ (i.e. the updated fixed point values for the other companies when 2's bid is $\left.b_{2}\left(v_{1}, v_{3}\right)=v_{2}^{\prime}\right)$. We have $v_{1}^{*}>v_{3}^{*}$, since $v_{1}^{*}=b_{1}\left(v_{2}^{\prime}, v_{3}^{*}\right)=50+0.3 v_{2}^{\prime}+0.5 v_{3}^{*}$ and $v_{3}^{*}=b_{3}\left(v_{2}^{\prime}, v_{1}^{*}\right)=70$, and so $v_{1}^{*}=85+0.3 \max \left\{v_{1}^{*}, v_{3}^{*}\right\}$. Solving, $v_{2}^{\prime}=v_{1}^{*}$ and $v_{1}^{*}=850 / 7 \approx 121.4$ solves $v_{1}^{*}=85+0.3 v_{1}^{*}$. Thus, company 2 wins for a price of 121.4. Notice that this is less than $v_{1}^{\circ}$, since the contingent value of company 1 is also reduced in determining the threshold bid at which 2 would have just won.

\subsection{Establishing Technical Conditions}

Given the linear bidding language we must show the uniqueness of a fixed point, and then also monotonicity and SCC. First, we prove conditions on weights $\alpha_{i j}$ for which the fixed point of the mapping $\left(v_{1}, \ldots, v_{N}\right) \mapsto\left(b_{1}\left(v_{-1}\right), \ldots, b_{N}\left(v_{-N}\right)\right)$ is unique. For this we adopt Banach's Fixed Point Theorem (see Vohra [17]).

TheOrem 3 (BANACH's FiXed PoInt TheOrem). $(B F P T)$ Let $(Y, d)$ be a complete metric space 4 and let $f: Y \rightarrow Y$

\footnotetext{
${ }^{4}$ A distance $d$ is a metric for set $Y$ if it satisfies: $d(x, y)+d(y, z) \geq$
} 
be a contraction mapping on $Y$, i.e. such that there is some real number $q<1$ for which

$$
d(f(x), f(y)) \leq q \cdot d(x, y)
$$

for all $x, y \in Y$. Then, the function $f$ admits a unique fixed point $x^{*}$, which can be computed as the limit of applying $f$ from any arbitrary point $x_{0}$ in $Y$.

Note that BFPT requires a complete metric space but allows a metric space that is not compact. So, for $Y$ adopt the space of nonnegative valuations, i.e. $\mathbf{R}_{\geq 0}^{N}$ for $N$ bidders. Instantiate mapping $f$ as $b(v)=\left(b_{1}\left(v_{-1}\right), \ldots, b_{N}\left(v_{-N}\right)\right)$, where $b_{i}$ is the contingent bid function, Eq. (6). This is a function into $\mathbb{R}_{\geq 0}^{N}$. Define the distance metric, $d$, as:

$$
d(v, w)=\max _{i \in\{1, \ldots, N\}}\left|v_{i}-w_{i}\right|,
$$

for $v, w \in \mathbb{R}_{>0}^{N}$. For example, consider two agents with $\alpha_{12}=$ $\alpha_{21}=0.5$, and consider values $v=\left(v_{1}, v_{2}\right) \in \mathbf{R}_{\geq 0}^{2}$ and $w=$ $\left(w_{1}, w_{2}\right) \in \mathbf{R}_{>0}^{2}$. Then, we have $d(v, w)=\max \left(\left|v_{1}-w_{1}\right|, \mid v_{2}-\right.$ $\left.w_{2} \mid\right)$ and $d(b(v), b(w))=\max \left(\left|s_{1}+v_{2} / 2-\left(s_{1}+w_{2} / 2\right)\right|, \mid s_{2}+\right.$ $\left.v_{1} / 2-\left(s_{2}+w_{1} / 2\right) \mid\right)=\max \left(\left|v_{1} / 2-w_{1} / 2\right|,\left|v_{2} / 2-w_{2} / 2\right|\right)$. Clearly, $\max \left(\left|v_{1} / 2-w_{1} / 2\right|,\left|v_{2} / 2-w_{2} / 2\right|\right)<\max \left(\mid v_{1}-\right.$ $\left.w_{1}|,| v_{2}-w_{2} \mid\right)$. This analysis extends to the general case.

LEMMA 1. There is a unique valuation equilibrium when $\sum_{j \neq i} \alpha_{i j}<1$ for all bidders $i$, and this equilibrium can be computed as the limit to a sequence of the contingent-bid mapping $b: \mathbb{R}_{\geq 0}^{N} \rightarrow \mathbb{R}_{\geq 0}^{N}$, starting from an arbitrary $v^{0} \in \mathbf{R}_{\geq 0}^{N}$.

Proof. Fix some $v, w \in \mathbb{R}_{>0}^{N}$. To show is $\max _{i}\left\{\mid b_{i}(v)-\right.$ $\left.b_{i}(w) \mid\right\}<\max _{i}\left\{\left|v_{i}-w_{i}\right|\right\}$. Consider any agent $i$. We will establish $\left|b_{i}(v)-b_{i}(w)\right|<\max _{j \neq i}\left\{\left|v_{j}-w_{j}\right|\right\}$, which implies the required result. Expanding, we require $\mid s_{i}+\sum_{j \neq i} \alpha_{i j} v_{j}-\left(s_{i}+\right.$ $\left.\sum_{j \neq i} \alpha_{i j} w_{j}\right) \mid<\max _{j \neq i}\left\{\left|v_{j}-w_{j}\right|\right\}$. Simplifying, we require $\left|\sum_{j \neq i}\left(v_{j}-w_{j}\right)\right|<\max _{j \neq i}\left\{\left|v_{j}-w_{j}\right|\right\}$. For this, it is sufficient to show that $\sum_{j \neq i} \alpha_{i j}\left|v_{j}-w_{j}\right|<\max _{j \neq i}\left\{\left|v_{j}-w_{j}\right|\right\}$, which is true since $\sum_{j \neq i} \alpha_{i j}<1$ and $\alpha_{i j} \in[0,1)$.

Thus, for uniqueness it is sufficient that the language restricts the total weight that each bidder $i$ assigns to the values of other bidders so that $\sum_{j \neq i} \alpha_{i j}<1$. For truthful bidding to form an ex post Nash equilibrium we also need the analogs to monotonicity and SCC to hold in the contingent-bid model.

LEMMA 2. For all contingent bids with $\sum_{j \neq i} \alpha_{i j}<1$ for all bidders $i$, then $\partial v_{j}^{\circ} / \partial v_{i 0}<\partial v_{i}^{\circ} / \partial v_{i 0}$ for all bidders $i, j$ (i.e. the analog to the single-crossing condition).

Proof. Consider some finite number of agents, $N$. Adopt $w_{i}$ to denote $v_{i 0}$ in agent bids. Towards a contradiction, assume

$$
\frac{\partial v_{1}^{\circ}}{\partial w_{1}} \leq \frac{\partial v_{2}^{\circ}}{\partial w_{1}}=\alpha_{21} \frac{\partial v_{1}^{\circ}}{\partial w_{1}}+\sum_{j \neq\{1,2\}} \alpha_{2 j} \frac{\partial v_{j}^{\circ}}{\partial w_{1}}
$$

Since $\sum_{j \neq 1} \alpha_{1 j}<1$ and $\alpha_{1 j} \geq 0$ this implies $N>2$ and $\partial v_{j}^{\circ} / \partial w_{1}>\partial v_{2}^{\circ} / \partial w_{1}$ for some $j \neq\{1,2\}$. W.l.o.g., suppose that $\overline{d(x, z), d(x, y)=d(y, x)}, d(x, x)=0$, and $d(x, y)=0 \Rightarrow x=$ $y$ for all $x, y, z \in Y$. A metric space is complete if it contains all limit points of Cauchy sequences, where a Cauchy sequence is a sequence in which $\lim _{m \rightarrow \infty} d\left(x_{m}, x_{m+1}\right)=0$. E.g., $(0,1)$ is not complete because $1 / 2,1 / 3, \ldots$ is Cauchy but does not have a limit in the space. On the other hand, [0, 1] is complete, as are $\mathbf{R}^{d}$ and $\mathbf{R}_{\geq 0}^{d}$. A complete metric space need not be closed (or compact). $\partial v_{3}^{\circ} / \partial w_{1}>\partial v_{2}^{\circ} / \partial w_{1} \geq \partial v_{1}^{\circ} / \partial w_{1}$. Considering agent 3 , this requires

$$
\alpha_{31} \frac{\partial v_{1}^{\circ}}{\partial w_{1}}+\alpha_{32} \frac{\partial v_{2}^{\circ}}{\partial w_{1}}+\sum_{j \neq\{1,2,3\}} \alpha_{3 j} \frac{\partial v_{j}^{\circ}}{\partial w_{1}}>\frac{\partial v_{2}^{\circ}}{\partial w_{1}}
$$

Thus, we must have $N>3$ agents. W.l.o.g., suppose that $\partial v_{4}^{\circ} / \partial w_{1}>\partial v_{3}^{\circ} / \partial w_{1}$. This induction continues indefinitely, leading to a contradiction for any finite number of agents.

Informally, the system of equations cannot sustain a fixed point at which the dependency of some $v_{j}^{\circ}$, for $j \neq i$, on $v_{i 0}$ is greater than that of $v_{i}^{\circ}$ itself because the effect is "dampened" in all bids by some multiplier less than one. Monotonicity, which requires $v_{i}^{\circ} / \partial v_{i 0} \geq 0$ is immediate since all coefficients in the mapping $v \mapsto b(v)$ are non-negative.

THEOREM 4. The contingent-bid auction defined with the linear contingent-bid language is efficient (in an ex post Nash equilibrium) when the language is expressive.

By "expressive", we mean that a bidder should be able to use the language to define a bid value that is equal to its true value, whatever the values of the other bidders. The proof follows from Lemmas 1 and 2, together with Theorem 2. Informally, once uniqueness is ensured so that the auction is well defined, fix the bids $b_{-i}$ from agents $j \neq i$. Agent $i$ faces an agent-independent price, defined in terms of the smallest uncontingent bid $b_{i}^{\prime}\left(v_{-i}\right)=v_{i 0}^{\prime}$ at which it would win in the valuation equilibrium, given bids $b_{-i}$. Then, by SCC and monotonicity, and assuming the other agents are bidding truthfully, the agent will win (and make this payment) when it bids truthfully whenever its true value is greater than this price and not otherwise. Realize that an agent cannot gain by misstating either its stand-alone value $v_{i 0}$ or its $\alpha$-weights.

\section{TOWARDS REVENUE OPTIMALITY}

We can consider a family of modified interdependent value auctions to find an auction with improved revenue. Following the direction set by Likhodedov and Sandholm [12] (in private value combinatorial auctions), we introduce a "dummy agent" (indexed zero) to represent the seller and then modify the winnerdetermination problem by defining static weights $\beta_{i} \in[0,1]$ on each agent. An allocation $x=\left(x_{0}, x_{1}, \ldots, x_{N}\right)$, with $x_{i} \in\{0,1\}$, $x_{0} \in\{0,1\}$ to indicate whether the dummy agent receives the item and the item goes unsold, and $\sum_{i=0}^{N} x_{i}=1$, is selected to solve:

$$
\max _{x}\left\{\beta_{0} v_{0}(x)+\sum_{i=1}^{N} \beta_{i} v_{i}^{\circ}(x)\right\},
$$

where $v_{i}^{\circ}(x)=v_{i}^{\circ}$ if $x_{i}=1$ and 0 otherwise, $v_{0}(x)=v_{0}$ if $x_{0}=1$ and 0 otherwise denotes the value of the dummy agent ( $v_{0}$ can be thought of as the reservation price), and $v_{i}^{\circ}$ are agent's fixed point values.

Value $v_{0}$ and weights $\beta$ are fixed before bids are received and heuristically optimized by the auction designer using prior information about agent values. The weighted contingent-bid auction is defined as:

1. Define $v_{0}$ and weights $\beta \in[0,1]^{N+1}$.

2. Receive bids and compute the fixed point $\left(v_{1}^{\circ}, \ldots, v_{N}^{\circ}\right)$. (Note that agents cannot make their bids contingent on the dummy agent's value, and that the dummy agent's value is uncontingent and fixed at $v_{0}$.) 


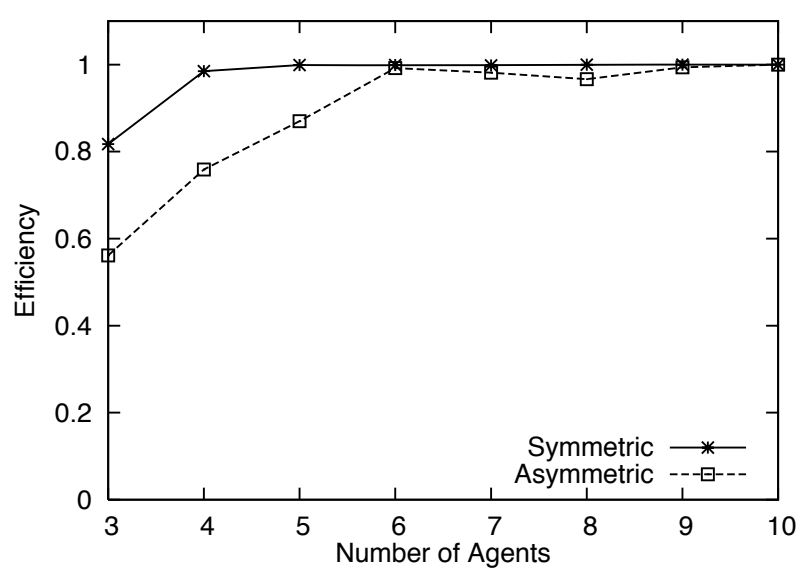

Figure 1: Average Efficiency in the Optimal Auction for the Symmetric and Asymmetric Environments.

3. Compute the winner as the agent with the maximal weighted value. If the dummy agent wins then the good is not sold and no revenue is received.

4. If the item is sold to agent $i \neq 0$, then compute the payment: Decrease $v_{i}^{\prime}$ until $\beta_{i} v_{i}^{\prime}=\max _{j \neq i, j=0}\left\{\beta_{j} v_{j}^{*}\right\}$, where $v_{j}^{*}$ is the new fixed point for agent $j$ given value $v_{i}^{\prime}$ to agent $i$ (i.e., $v_{j}^{*}=b_{j}\left(v_{i}^{\prime}, v_{-i j}^{*}\right)$ for all $j$ ). This value, at which $i$ would be just about to stop winning, is the payment.

Clearly, this auction reduces to the standard auction when the weights are symmetric and $v_{0}=0$. From Myerson's [15] work on optimal auctions, we should expect weights that bias in favor of agents that are a priori less competitive. It should also be fairly clear that the auction remains truthful. One needs to show that the price to the winner is still independent of its bid, and that the winner wins when its fixed point valuation is above the price, and only then. (The proof is omitted in the interest of space.)

In our experiments, we consider a symmetric environment with the signal $s_{i}$ for each agent $i \neq 0$ is sampled $s_{i} \sim$ $\operatorname{Uniform}(0,100)$, and an asymmetric environment with the signal for agent $i$ is sampled from distribution $\operatorname{Uniform}\left(0, L_{i}\right)$, where $L_{i}=10+i \delta$ and $\delta=\frac{300-10}{N}$. This has the effect of making the expected signal value of agents increase with the index of the agent in the asymmetric environment. Then, for each agent $i$ we determine the $\alpha$-weights that define the contingent valuations as follows:

(1) Each $j \neq i$ has $\alpha_{i j}=0$ with prob. 0.5 , otherwise sample $\alpha_{i j}$ from Uniform $(0,1)$.

(2) Determine a total alpha-weight, $m_{i}$, as follows:

(3.1) For the symmetric environment, by sampling from a Gaussian distribution with mean 0.5 and standard deviation 0.25 , truncated to $[0,1)$.

(3.2) For the asymmetric environment, by sampling from a Gaussian distribution with mean $\mu_{i}=0.3+i \delta^{\prime}$ and standard deviation 0.25 , truncated to $[0,1)$, with $\delta^{\prime}=\frac{1.0-0.3}{N}$. The effect is to cause the expected total weight assigned to the values of other agents to increase with the index of the agent.

(4) Normalize weights $\alpha_{i}$ to have total weight $m_{i}$, by multiplying each $\alpha_{i j}$ by $\frac{m_{i}}{\sum_{j \neq i} \alpha_{i j}}$.

We use search over auction parameters $\left(v_{0}, \beta_{0}, \beta_{1}, \ldots, \beta_{N}\right)$, to maximize the expected revenue. In all cases, we normalize weights

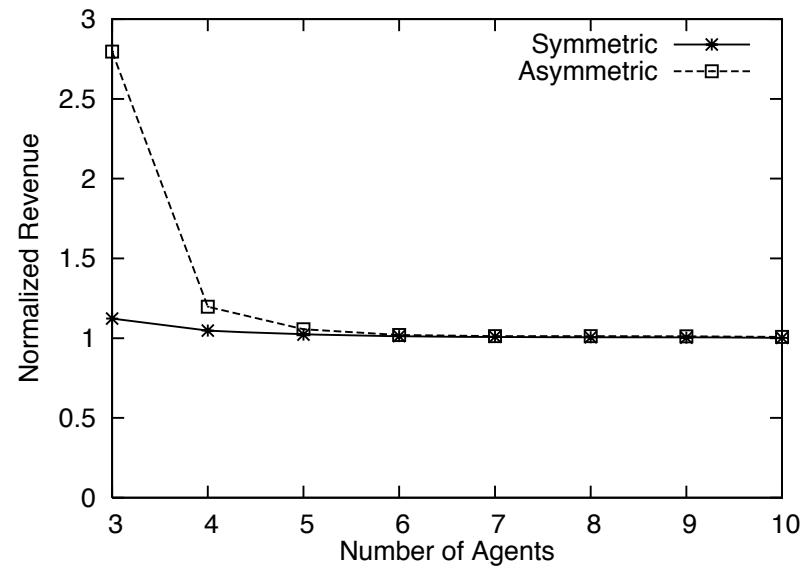

Figure 2: Average Normalized Revenue in the Optimal Auction for the Symmetric and Asymmetric Environments.

so that $w_{0}=1 / N$ and $\sum_{i=1}^{n} w_{i}=1$, so that $\sum_{i=0}^{n}=(1+$ $N) / N$. Normalization is achieved by setting weights on $i>1$ and making $w_{1}$ a dependent variable. The search space is discretized, with $v_{0} \in\{0,10, \ldots, 100\}$, and weights $w_{i} \in\{0,0.1, \ldots, 1\} \cup$ $\{1 / N\}$. Because our problem was quite small and because our goal was to get an initial understanding of whether revenue can be improved in interdependent value auctions we simply enumerate all grid points and select the optimal auction parameters. Future work will explore local search and other approximation methods, as in Likhodedov and Sandholm [13].

To evaluate a parameterization, we average over 4000 instances for the symmetric environment and 5000 instances for the asymmetric environment. We compare the average revenue and efficiency in the optimized auctions with the revenue and efficiency of the standard contingent bid auctions.

Figure 1 illustrates the average efficiency of the optimal auction, i.e. the average ratio of total value in the optimal auction to the maximal value (achieved in the standard auction), for different numbers of agents. Figure 2 illustrates the average normalized revenue of the optimal auction, i.e. the average ratio of total revenue in the optimal auction to the total revenue in the efficient auction, for different numbers of agents. As expected, we see that the auctions can be optimized for revenue, although at some loss in efficiency and with the benefit quickly dropping off as the number of agents increases to 5 or more. In addition, for our distributions, the possible benefit is greater in the asymmetric setting than in the symmetric setting.

Looking at the optimal parameters, the optimal revenue was achieved in the symmetric environment with symmetric weights. For the asymmetric environment, the optimal weights were sometimes asymmetric, for instance when there are 3 agents the optimal weight assignment was 0.5 for agent $1,0.3$ for agent 2 , and 0.2 for agent 3. Moreover, optimal weights were always decreasing with agents as we expected from Myerson's [15] study of asymmetric private value auctions. This suggests that for local search it would be useful to restrict the search space to only allow states with weights that are (weakly) decreasing with agent index. The optimal choice for $v_{0}$ in the asymmetric environment varied from 50 to 110 , and averaged around 80 .

\section{SINGLE-MINDED CAS}

Many interesting auction scenarios have multiple items and inter- 
dependent values. For instance, consider selling a mix of different bottles and allowing bidders to put together there own "cases" of wine. By adopting single-minded bidders, interested in a particular bundle of goods, we consider the simplest possible model of interdependent value CAs. Agents have two-dimensional, consisting of the description of the bundle in which it is interested and its signal, conveying information about the value of its bundle (and perhaps about the value of other agents for other bundles.) We construct an approximately efficient auction, which satisfies a generalization of the single-crossing condition.

\subsection{Extending the Contingent-Bid Language}

Let $G$ denote the set of goods. A single-minded bidder has an interesting bundle $W_{i} \subseteq G$ and an interdependent valuation function $z_{i}(X, s) \geq 0$ on bundles $X \subseteq G$, defined in terms of signals $s=\left(s_{1}, \ldots, s_{N}\right)$ as:

$$
z_{i}(X, s)= \begin{cases}z_{i}(s) & \text { if } X \supseteq W_{i} \\ 0 & \text { otherwise }\end{cases}
$$

Here, we assume free-disposal, so that an agent's value is (weakly) increasing with additional goods. Function, $z_{i}(s) \geq 0$, defines the agent's value for an interesting bundle given signals $s$.

The contingent-bid language is extended to allow a bid by agent $i$ to report an interesting bundle, $\hat{W}_{i}$ (perhaps untruthfully), a stand-alone value $v_{i 0} \geq 0$, and weight-bundle pairs $\left\{\left(\alpha_{i 1}, X_{i 1}\right), \ldots,\left(\alpha_{i N}, X_{i N}\right)\right\}$ for agents $j \neq i$. Taken together, this defines the following contingent bid function:

$$
b_{i}\left(X, v_{-i}\right)= \begin{cases}v_{i 0}+\sum_{j \neq i} \alpha_{i j} v_{j}\left(X_{i j}\right), & \text { for } X \supseteq \hat{W}_{i} \\ 0, & \text { otherwise }\end{cases}
$$

We follow the earlier notation: $b_{i}$ is a contingent-bid, now for each bundle $X$, and $v_{i}(X)$ denotes an agent's value for a bundle (determined either in a fixed point, or via its valuation function $z_{i}$ and signals of others). We also require $\alpha_{i j} \in[0,1)$ and $\sum_{j \neq i} \alpha_{i j}<1$ for all $i$, as before. The valuation equilibrium is defined as a fixed point of the mapping $\left(v_{1}, \ldots, v_{N}\right) \mapsto$ $\left(b_{1}\left(v_{-1}\right), \ldots, b_{N}\left(v_{-N}\right)\right)$, where $v_{i}: 2^{G} \rightarrow \mathbb{R}_{\geq 0}$ and $b_{i}\left(v_{-i}\right):$ $2^{G} \rightarrow \mathbb{R}_{\geq 0}$ (i.e. the restriction of the agent's contingent bid function, given values of other agents for each bundle.)

An agent's value for some bundle $X$ is defined in terms of the value of other agents $j \neq i$ for other bundles $X_{i j}$, and also depends on whether $X \supseteq \hat{W}_{i}$. Realize that $X_{i j}$, the bundle that bidder $i$ picks out for bidder $j$, in defining its dependency on the valuation function of bidder $j$, need not be the same as either $\hat{W}_{i}$ or $\hat{W}_{j}$. This provides useful expressiveness as well as ensuring that the knowledge requirements are reasonable. (See the example in Section 5.3.)

THEOREM 5. The single-minded contingent bid model has a unique fixed point when $\sum_{j \neq i} \alpha_{i j}<1$ for all $i$.

PROOF. By reduction to the single-item contingent bid model. Given a set of contingent bids $b$, simplify the bids as follows: for each agent $i$, replace $X_{i j}$ with $\hat{W}_{j}$ if $X_{i j} \supseteq \hat{W}_{j}$ and replace $X_{i j}$ with $\emptyset$ otherwise. Since $v_{i}(\emptyset)=0$ for all agents, this has the effect of removing any dependence on bundles of this kind. Given this simplification, the fixed point can be characterized as the fixed point to the system of equations

$$
\begin{gathered}
b_{1}\left(\hat{W}_{i}, v_{-1}\right)=v_{10}+\sum_{j \neq 1} \alpha_{1 j} v_{j}\left(\hat{W}_{j}\right) \\
\ldots \\
\ldots \\
b_{N}\left(\hat{W}_{N}, v_{-N}\right)=v_{N 0}+\sum_{j \neq N} \alpha_{N j} v_{j}\left(\hat{W}_{j}\right),
\end{gathered}
$$

which have exactly the same form as for the single-item contingent bid model because the same bundle $\hat{W}_{i}$ appears for agent $i$ in all equations. Thus, there is a unique solution.

\subsection{Failure of Generalized SCC}

It is tempting to generalize the efficient auction described for the single item case as follows: (1) compute the fixed point valuation functions $v^{\circ}$ defined by the mapping induced by Eq. (16); (2) compute the efficient allocation $X^{*}=\left(X_{1}^{*}, \ldots, X_{N}^{*}\right)=$ $\arg \max _{X \in \Gamma} \sum_{i} v_{i}^{\circ}\left(X_{i}\right)$ to maximize total value (breaking ties at random), where $\Gamma$ denotes the feasible allocations (restricted to allocations in which agent $i$ either receives $\emptyset$ or $\hat{W}_{i}$ ); and (3) compute payment to each winner $i$, as:

$$
\begin{array}{cc}
\min & v_{i}^{\prime} \\
\text { s.t. } & v_{i}^{\prime}+\sum_{j \neq i} v_{j}^{*}\left(X_{j}^{*}\right) \geq \max _{X \in \Gamma} \sum_{j \neq i} v_{j}^{*}\left(X_{j}\right),
\end{array}
$$

where $v_{j}^{*}(X)=b_{j}\left(X,\left(\vec{v}_{i}^{\prime}, v_{-i j}^{*}\right)\right)$, and $\vec{v}_{i}^{\prime}(X)=v_{i}^{\prime}$ for $X \supseteq \hat{w}_{i}$ and zero otherwise. This has the same structure as the previous auction: $v_{-i}^{*}$ denotes the revised fixed point when agent $i$ bids value $v_{i}^{\prime}$ on its interesting bundle $\hat{W}_{i}$; thus, agent $i$ pays the smallest amount it could have bid and still won, also accounting for the effect of its new report on the values of other agents.

For truthful bidding to be an ex post Nash equilibrium in this auction, it would be sufficient for the valuations to satisfy a generalized form of the monotonicity and single-crossing conditions, ${ }^{5}$ which combine as:

DEFINITION 1 (GENERALIZED SCC). If, for signals $\left(s_{1}, \ldots, s_{N}\right)$ there is a pair of allocations $X$ and $X^{\prime}$, tied for value, i.e.:

$$
\sum_{j=1}^{N} z_{j}\left(X_{j}, s\right)=\sum_{j=1}^{N} z_{j}\left(X_{j}^{\prime}, s\right)=\max _{X^{\prime \prime} \in \Gamma} \sum_{j} z_{j}\left(X_{j}^{\prime \prime}, s\right)
$$

then for every agent $i$ such that $X_{i} \neq X_{i}^{\prime}$, we require:

$$
\begin{gathered}
{\left[\frac{\partial}{\partial s_{i}} z_{i}\left(X_{i}, s\right)>\frac{\partial}{\partial s_{i}} z_{i}\left(X_{i}^{\prime}, s\right)\right]} \\
\Longleftrightarrow\left[\frac{\partial}{\partial s_{i}} \sum_{j=1}^{N} z_{j}\left(X_{j}, s\right)>\frac{\partial}{\partial s_{i}} \sum_{j=1}^{N} z_{j}\left(X_{j}^{\prime}, s\right)\right]
\end{gathered}
$$

In words, generalized SCC requires that the value of agent $i$ for $X$ is improving more quickly than for $X^{\prime}$ with respect to its signal whenever this is true for the economy of agents in aggregate, and vice versa. Conceptually, this will be true when the marginal effect of an agent's signal on its own value dominates the marginal effect of its signal on the aggregate economy.

THEOREM 6. [14] The second-price interdependent value single-minded CA auction is efficient in an ex post Nash equilibrium when the generalized SCC property holds.

For private value single-minded CAs, this auction is equivalent to the VCG mechanism and truthful bidding is a dominant strategy equilibrium. But, we will show that generalized SCC, and thus incentive compatibility, fails in our interdependent value model. Consider Eq. (21) for single-minded CAs. The LHS of the bidirectional condition is only true when $X_{i}=W_{i}$ and $X_{i}^{\prime}=\emptyset$. We see that generalized SCC requires that whenever an allocation in

\footnotetext{
${ }^{5}$ Personal communication [14]. Earlier conditions, as described in Dasgupta and Maskin [3], appear too strong to be useful.
} 
which agent $i$ wins and an allocation in which agent $i$ loses are tied for total value, then if a change in agent $i$ 's signal changes the allocation it must be that an increase in its signal causes the allocation in which the agent is the winner to be selected. Thus, generalized SCC reduces to the following requirement: for any signals $s_{-i}$ of other agents, if an agent $i$ is a winner for some signal $s_{i}$ then the agent must remain a winner for all higher signals. Notice that the allocation of items to other agents can change for higher signals, as long as agent $i$ always retains bundle $W_{i}$.

\subsection{Example}

Consider goods $G=\{A, B, C\}$ and 5 agents, with (truthful) contingent bids:

$$
\begin{aligned}
b_{1}\left(A B, v_{-1}\right) & =10+0.5 v_{2}(C) \\
b_{2}\left(C, v_{-2}\right) & =10+0.4 v_{1}(A) \\
b_{3}\left(A B C, v_{-3}\right) & =5+0.2 v_{1}(A B)+0.6 v_{2}(A B C) \\
b_{4}\left(A, v_{-4}\right) & =1+2 / 3 v_{1}(A B C) \\
b_{5}\left(B C, v_{-5}\right) & =1+2 / 3 v_{1}(A B C)
\end{aligned}
$$

This can be simplified, replacing $X_{i j}$ with $W_{j}$ if $X_{i j} \supseteq W_{j}$ and $\emptyset$ otherwise (and removing this term.) We are left with $b_{2}\left(C, v_{-2}\right)=10+0.4 v_{1}(\emptyset)=10, b_{1}\left(A B, v_{-1}\right)=10+$ $0.5 v_{2}(C)=15, b_{3}(A B C)=14$, and $b_{4}(A)=b_{5}(B C)=11$.

Suppose agents bid truthfully with $b_{i}=v_{i}$ for all agents. The final allocation is $(A B, C, \emptyset, \emptyset, \emptyset)$. To compute the payment by agent 1 , we solve (by Eq. 19) $\min v_{1}^{\prime}$ s.t. $v_{1}^{\prime}+10 \geq 5+0.2 v_{1}^{\prime}+6$ (for allocation $(\emptyset, \emptyset, A B C, \emptyset, \emptyset)$ ), $v_{1}^{\prime}+10 \geq 10+1+2 / 3 v_{1}^{\prime}$ (for allocation $(\emptyset, C, \emptyset, A, \emptyset)$, and $v_{1}^{\prime}+10 \geq 1+2 / 3 v_{1}^{\prime}+1+2 / 3 v_{1}^{\prime}$ (for allocation $(\emptyset, \emptyset, \emptyset, A, B C)$. The first two constraints require $v_{1}^{\prime} \geq 1.25, v_{1}^{\prime} \geq 3$, while the third constraint not binding. Thus, agent 1's payment is $p_{1}=3$. At a bid equal to this price, the auctioneer would be indifferent between allocation $(A B, C, \emptyset, \emptyset, \emptyset)$ and $(\emptyset, C, \emptyset, A, \emptyset)$. For agent 2 , we solve $\min v_{2}^{\prime}$ s.t. $10+0.5 v_{2}^{\prime}+$ $v_{2}^{\prime} \geq 5+0.2\left(10+0.5 v_{2}^{\prime}\right)+0.6 v_{2}^{\prime}$ (allocation $\left.(\emptyset, \emptyset, A B C, \emptyset, \emptyset)\right)$ and $10+0.5 v_{2}^{\prime}+v_{2}^{\prime} \geq 2+4 / 3\left(10+v_{2}^{\prime} / 2\right)$ (allocation $(\emptyset, \emptyset, \emptyset, A, B C)$. Only the second constraint is binding, and requires $v_{2}^{\prime} \geq 32 / 5$. Thus, agent 2's payment is $32 / 5=6.4$.

To see the failure of generalized SCC, let $w_{1}$ denote agent 1 's stand-alone value and consider what happens as $w_{1}$ is increased from 10 . When $w_{1}=19$ we have $v_{1}\left(A B, v_{-1}\right)=19+0.5 v_{2}(C)$, and solving for the valuation equilibrium we find $v_{1}(A B)=$ $24, v_{2}(C)=10, v_{3}(A B C)=79 / 5=15.8, v_{4}(A)=17$ and $v_{5}(B C)=17$. At this point, allocations $X=(A B, C, \emptyset, \emptyset, \emptyset)$ and $X^{\prime}=(\emptyset, \emptyset, \emptyset, A, B C)$ are tied for value. In addition, $\partial v_{1}^{\circ}\left(X_{1}\right) / \partial w_{1}>\partial v_{1}^{\circ}\left(X_{1}^{\prime}\right) / \partial w_{1}=0$. For the analog to generalized SCC in the contingent-bid model, we need:

$$
\frac{\partial}{\partial w_{1}}\left[v_{1}^{\circ}\left(X_{1}\right)+v_{2}^{\circ}\left(X_{2}\right)\right]>\frac{\partial}{\partial w_{1}}\left[v_{4}^{\circ}\left(X_{4}^{\prime}\right)+v_{5}^{\circ}\left(X_{5}^{\prime}\right)\right],
$$

but this does not hold because $\partial\left(v_{1}^{\circ}\left(X_{1}\right)+v_{2}^{\circ}\left(X_{2}\right)\right) / \partial w_{1}=1<$ $\partial\left(v_{4}^{\circ}\left(X_{4}^{\prime}\right)+v_{5}^{\circ}\left(X_{5}^{\prime}\right)\right) / \partial w_{1}=4 / 3$.

The implication of this failure of generalized SCC is that if agent 1 has true value greater than 34 , and the other contingent valuations of agents were unchanged its equilibrium strategy is not to bid truthfully. For example, if its stand-alone value was 20 but its $\alpha$-weights were unchanged from the example, and if the bids $b_{-1}$ were unchanged, then the agent would lose if bidding truthfully. The selected allocation would give $A$ to agent 4 and $B C$ to agent 5. The agent could do better by reporting an uncontingent bid of $b_{1}\left(A B, v_{-1}\right)=15$; it would win and make a payment of 3 .

Failure of generalized SCC occurs in this example because agent 1 's value does not affect the value of the other agent (agent 2) with which it must form a winning coalition. On the other hand, its value affects the value of both agents 4 and 5 in the competing coalition.

\subsection{A Truthful Single-Minded CA}

We can instead define an approximately efficient auction using a greedy allocation rule, which is parameterized with some constant $c>0$. The auction generalizes the auction proposed in Lehmann et al. [11] for single-minded bidders and private values to the setting of interdependent values. ${ }^{6}$ It also reduces to our single-item auction when all agents bid on bundles with overlapping goods, so that only one agent can win. The auction is defined as follows:

1. Compute fixed point values $\left(v_{1}^{\circ}, \ldots, v_{N}^{\circ}\right)$ on reported bundles bundles $\left(\hat{W}_{1}, \ldots, \hat{W}_{N}\right)$.

2. Sort bids in decreasing order of $v_{i}^{\circ} /\left|\hat{W}_{i}\right|^{c}$, breaking ties at random. Constant $c>0$ determines the dependence of bid rank on the number of items in the interesting bundle. Walk down the list, accepting bids if the goods demanded are still unallocated. This is the greedy allocation rule.

3. Let $g_{i}^{c}\left(v^{\circ}\right) \in\{0,1\}$ denote whether agent $i$ is allocated its interesting bundle in the greedy allocation, given fixed point values $v^{\circ}$. For a winner, with $g_{i}^{c}\left(v^{\circ}\right)=1$, define the payment as the $\min v_{i}^{\prime}$ s.t. $g_{i}^{c}\left(\left(v_{i}^{\prime}, v_{-i}^{*}\right)\right)=1$ where $v_{-i}^{*}$ denotes the new fixed point given (uncontingent) bid $b_{i}\left(X, v_{-i}\right)=v_{i}^{\prime}$ on $X \supseteq \hat{W}_{i}$ and 0 otherwise.

LEMMA 3. For all contingent bids with $\sum_{j \neq i} \alpha_{i j}<1$ for all bidders $i$, the greedy allocation rule satisfies the generalized singlecrossing condition (with stand-along value $v_{i 0}$ in the role of signal $s_{i}$.).

PROOF. (sketch) From Lemma 2 we have $\frac{\partial}{\partial v_{i 0}} v_{j}^{\circ}\left(\hat{W}_{j}\right)<$ $\frac{\partial}{\partial v_{i 0}} v_{i}^{\circ}\left(\hat{W}_{i}\right)$, for all $j \neq i$. So, if agent $i$ is winning for some stand-alone value, $v_{i 0}$, then it will continue to win for a higher signal because its value will increase by more than the value of any other agent and therefore its rank in the ordered list of bids to accept can only improve.

The greedy rule recovers the generalized SCC requirement because agent $i$ must only compete unilaterally with each other agent that demands one or more of the same goods. By comparison, the efficient allocation rule considered competition between coalitions of agents in deciding whether agent $i$ was a winner.

THEOREM 7. The greedy contingent-bid auction for singleminded bidders and multiple items supports truthful bidding in an ex post Nash equilibrium when the bidding language is expressive. Moreover, when parameter $c=1 / 2$ the greedy auction is $\sqrt{m}$-competitive w.r.t. efficiency, for $m$ goods.

We omit the proof of equilibrium in the interest of space, and because it is in all essential aspects the same as that of the singleitem contingent bid auction. Truthfulness follows from the property of generalized SCC (Lemma 3). The worst-case approximation of $\sqrt{m}$ for the greedy rule with ordering metric $v_{i}^{\circ} /\left|W_{i}\right|^{1 / 2}$ follows immediately from Lehmann et al. [11]. The average case efficiency may be much better.

\footnotetext{
${ }^{6}$ Lehmann et al. [11] adopted the greedy allocation rule because the efficient winner-determination problem is NP-hard. Here, we adopt the greedy allocation rule because the efficient allocation rule is not implementable in an ex post Nash equilibrium when agents have interdependent values. Of course, computational complexity also provides another reason to prefer this rule in interdependent value problems with many bids.
} 


\subsection{Example: Truthful Greedy Auction}

For this example, adopt $c=1$ so that the rank of bids is determined by computing the per-item bid value. Consider the greedy auction on the earlier example, where we have $v_{1}^{\circ}(A B)=$ $15, v_{2}^{\circ}(C)=10, v_{3}^{\circ}(A B C)=14, v_{4}^{\circ}(A)=11$ and $v_{5}^{\circ}(B C)=$ 11. The auction assigns adjusted values $15 / 2,10,14 / 3,11$ and $11 / 2$ to each bid (i.e. the per-item bid values), and places the bids in decreasing order as bids $4,2,1,5$ and 3 . Agent 4 is allocated $A$, agent 2 is allocated $C$, and then no other agents receive any goods because one or more items in their interesting bundles are already allocated. The allocation is $(\emptyset, C, \emptyset, A, \emptyset)$. (Note that this is not the efficient allocation.) Agent 2's payment is determined by considering the values of bids that it displaces, namely the bids from agents 3 and 5. (It must simply remain ranked above agents 3 and 5 to remain a winner.) Considering agent 3 , the constraint is $v_{2}^{\prime} \geq 1 / 3\left(5+0.2\left(10+0.5 v_{2}^{\prime}\right)+0.6 v_{2}^{\prime}\right)$ (where the factor $1 / 3$ is included because agent 3 bids on 3 items, and thus this reduces its rank), which gives $v_{2}^{\prime} \geq 70 / 23$. Considering agent 5 , the constraint is $v_{2}^{\prime} \geq 1 / 2\left(1+2 / 3\left(10+0.5 v_{2}^{\prime}\right)\right)$ (factor $1 / 2$ because agent 5 bids on 2 items), which reduces to $v_{2}^{\prime} \geq 23 / 5$. The latter constraint is binding, and agent 2's payment is $p_{2}=23 / 5=4.6$. Agent 4 displaces bids from agents 1 and 3 , and its payment is computed as $\min v_{4}^{\prime}$ s.t. $v_{4}^{\prime} \geq \max \{15 / 2,14 / 3\}=15 / 2=7.5$. $^{7}$

\section{CONCLUSIONS AND FUTURE WORK}

We have instantiated the contingent bid model of Dasgupta and Maskin [3] to provide a concrete protocol for interdependent value auctions. A simple linear bidding language is demonstrated to satisfy the technical conditions required for the efficiency of a contingent-bid second-price auction. Seeking good revenue properties, we also illustrated protocols with better expected revenue.

In addition, we described a protocol for interdependent-value combinatorial auctions (CAs) with single-minded bidders. Thus, we find a positive counterpoint to the negative general conclusions in the literature on interdependent value auctions with multidimensional private information $[8,9] .{ }^{8}$ An important future direction is to study other models that provide structure to value interdependencies and allow for similarly positive results.

In principle, a more general pricing method described in Dasgupta and Maskin [3] can be used to design truthful auctions in combinatorial auctions without the restriction to single-minded bidders. (As long as the generalized SCC condition continues to hold, and with single-dimensional private information.) However, we wish to draw attention to what we believe to be a severe practical limitation of the generalized method:

in addition to reporting contingent bids, agents are required to report the residual domain of valuations that it believes to be possible for other agents, given its own signal and given its knowledge about the functional form of the valuations of other agents.

This requirement, which is also discussed in Perry and Reny [16], is used to ensure that agent-independent payments can be computed that are consistent (in a utility-maximizing sense) with the efficient allocation. Requiring that every bidder reports

\footnotetext{
${ }^{7}$ It is instructive to consider the effect of agent 1 increasing its value in the greedy auction. Recall that this is where we observed the failure of generalized SCC in the proposed efficient auction. In order to win in the greedy auction, agent 1 must rank above agents 3 and 4 , of which agent 4 dominates. To rank above agent 4 , we need $v_{1}^{\prime} / 2 \geq 1+2 / 3 v_{1}^{\prime}$, which is impossible for $v_{i}^{\prime} \geq 0$. So, agent 1 can never win in this auction given the contingent bids of the other agents.

${ }^{8}$ See also Bikhchandani [1] for some additional possibility results.
}

exact and complete information on the residual valuation domains of other bidders seems untenable in practical systems. It seems likely to place a large burden on participants. We think future work should seek mechanisms for interdependent values that are (necessarily) inefficient when agents do not reveal adequate information, but fully efficient in the complete-revelation limit.

\section{Acknowledgments}

Parkes is supported in part by National Science Foundation grants IIS-0238147 and an Alfred P. Sloan Foundation award.

\section{REFERENCES}

[1] S. Bikhchandani. The limits of ex-post implementation revisited. Working Paper, UCLA, 2005.

[2] J. Cremer and R. P. McLean. Full extraction of surplus in bayesian and dominant strategy aucitons. Econometrica, 56:1247-1257, 1988.

[3] P. Dasgupta and E. Maskin. Efficient auctions. The Quarterly Journal of Economics, CXV:341-388, 2000.

[4] R. K. Dash, A. Rogers, and N. R. Jennings. A mechanism for multiple goods and interdependent valuations. In Proc. 6th Int. Workshop on Agent-Mediated E-Commerce, pages 197-210, 2004.

[5] T. Ito, M. Yokoo, and S. Matsubara. Designing an auction protocol under asymmetric information on nature's selection. In Proc. of the 1st International Joint Conference on Autonomous Agents and Multi-Agent Systems (AAMASO2), pages 61-68, 2002.

[6] T. Ito, M. Yokoo, and S. Matsubara. Towards a combinatorial auction protocol among experts and amateurs: The case of single-skilled experts. In Proc. of the 2nd International Joint Conference on Autonomous Agents and Multi-Agent Systems (AAMAS03), pages 481-488, 2003.

[7] T. Ito, M. Yokoo, and S. Matsubara. A combinatorial auction protocol among versatile experts and amateurs. In Proc. of the 3rd International Joint Conference on Autonomous Agents and Multi-Agent Systems (AAMASO4), pages 378-385, 2004.

[8] P. Jehiel and B. Moldovanu. Efficient design with interdependent valuations. Econometrica, 69(5):1237-1259, 2001.

[9] P. Jehiel, M. M. ter Vehn, B. Moldovanu, and W. R. Zame. The limits of ex-post implementation. Working Paper, 2005.

[10] V. Krishna. Auction Theory. Academic Press, 2002.

[11] D. Lehmann, L. I. O'Callaghan, and Y. Shoham. Truth revelation in rapid, approximately efficient combinatorial auctions. Journal of the ACM, 49:577-602, 2002.

[12] A. Likhodedov and T. Sandholm. Methods for boosting revenue in combinatorial auctions. In Proc. of the National Conference on Artificial Intelligence (AAAI), pages 232-237, 2004.

[13] A. Likhodedov and T. Sandholm. Approximating revenue-maximizing combinatorial auctions. In Proc. of the National Conference on Artificial Intelligence, pages 267-274, 2005.

[14] E. Maskin. Private communication, October 72005.

[15] R. B. Myerson. Optimal auction design. Mathematics of Operations Research, 6:58-73, 1981.

[16] M. Perry and P. J. Reny. An efficient auction. Econometrica, pages 1199-1212, 2002.

[17] R. Vohra. Advanced Mathematical Economics. Routledge, 2005. 